\title{
Solvability of fractional boundary value problems with $p$-Laplacian operator
}

\section{Bo Zhang*}

\section{Correspondence: \\ zhangbohuaibei@163.com \\ School of Mathematical Sciences, \\ Huaibei Normal University, Huaibei, \\ 235000, P.R. China}

\section{囪 Springer}

\begin{abstract}
This paper studies the existence of solutions for two boundary value problems for the fractional $p$-Laplacian equation. Under certain nonlinear growth conditions of the nonlinearity, two new existence results are obtained by using Schaefer's fixed point theorem.

MSC: 34A08; 34B15

Keywords: fractional differential equation; $p$-Laplacian operator; boundary value problem; Schaefer's fixed point theorem
\end{abstract}

\section{Introduction}

The fractional calculus is a generalization of the ordinary differentiation and integration on an arbitrary order that can be noninteger. In the last two decades, the theory of fractional calculus has gained importance and popularity due to its wide range of applications in varied fields of sciences and engineering. In [1-8], the applications are mentioned to fluid flow, rheology, dynamical processes in self-similar and porous structures, electrical networks, control theory of dynamical systems, viscoelasticity, electrochemistry of corrosion, chemical physics, optics and signal processing, and so on. Recently, many important results about the fractional differential equations have been given. For example, for fractional initial value problems, the existence and multiplicity of solutions were discussed in [4, 9-11]. In addition, for fractional boundary value problems (BVPs for short), Agarwal et al. (see [12]) considered a two-point BVP at nonresonance, and Bai (see [13]) considered an $m$-point BVP at resonance. For more papers on the fractional BVPs, see [14-22] and the references therein.

The turbulent flow in a porous medium is a fundamental mechanics problem. For studying this type of problems, Leibenson (see [23]) introduced the $p$-Laplacian equation as follows:

$$
\left(\phi_{p}\left(x^{\prime}(t)\right)\right)^{\prime}=f\left(t, x(t), x^{\prime}(t)\right)
$$

where $\phi_{p}(s)=|s|^{p-2} s, p>1$. Obviously, $\phi_{p}$ is invertible and its inverse operator is $\phi_{q}$, where $q>1$ is a constant such that $1 / p+1 / q=1$. In the past few decades, many important results about equation (1.1) with certain boundary value conditions have been obtained. We refer the readers to [24-31] and the references cited therein.

(c) 2015 Zhang. This article is distributed under the terms of the Creative Commons Attribution 4.0 International License (http://creativecommons.org/licenses/by/4.0/), which permits unrestricted use, distribution, and reproduction in any medium, provided you give appropriate credit to the original author(s) and the source, provide a link to the Creative Commons license, and indicate if changes were made. 
Motivated by the works mentioned previously, in this paper, we investigate the existence of solutions for the fractional $p$-Laplacian equation of the form

$$
D_{0^{+}}^{\beta} \phi_{p}\left(D_{0^{+}}^{\alpha} x(t)\right)=f\left(t, x(t), D_{0^{+}}^{\alpha} x(t)\right), \quad t \in[0,1]
$$

subject to the boundary value conditions either

$$
D_{0^{+}}^{\alpha} x(0)=x(1)=0
$$

or

$$
x(0)=x(1)=0,
$$

where $0<\alpha, \beta \leq 1, D_{0^{+}}^{\alpha}$ is a Caputo fractional derivative, and $f:[0,1] \times \mathbb{R}^{2} \rightarrow \mathbb{R}$ is continuous. Note that the nonlinear operator $D_{0^{+}}^{\beta} \phi_{p}\left(D_{0^{+}}^{\alpha}\right)$ reduces to the linear operator $D_{0^{+}}^{\beta} D_{0^{+}}^{\alpha}$ when $p=2$ and the additive index law

$$
D_{0^{+}}^{\beta} D_{0^{+}}^{\alpha} u(t)=D_{0^{+}}^{\alpha+\beta} u(t)
$$

holds under some reasonable constraints on the function $u$ (see [32]).

The rest of this paper is organized as follows. Section 2 contains some necessary notations, definitions and lemmas. In Section 3, based on Schaefer's fixed point theorem, we establish two theorems on the existence of solutions for BVP (1.2) (1.3) (Theorem 3.1) and BVP (1.2) (1.4) (Theorem 3.2). Finally, in Section 4, an explicit example is given to illustrate the main results.

\section{Preliminaries}

For convenience of the readers, we present here some necessary basic knowledge and definitions as regards the fractional calculus theory, which can be found, for instance, in [33, $34]$.

Definition 2.1 The Riemann-Liouville fractional integral operator of order $\alpha>0$ of a function $u:(0,+\infty) \rightarrow \mathbb{R}$ is given by

$$
I_{0^{+}}^{\alpha} u(t)=\frac{1}{\Gamma(\alpha)} \int_{0}^{t}(t-s)^{\alpha-1} u(s) d s
$$

provided that the right-hand side integral is pointwise defined on $(0,+\infty)$.

Definition 2.2 The Caputo fractional derivative of order $\alpha>0$ of a continuous function $u:(0,+\infty) \rightarrow \mathbb{R}$ is given by

$$
\begin{aligned}
D_{0^{+}}^{\alpha} u(t) & =I_{0^{+}}^{n-\alpha} \frac{d^{n} u(t)}{d t^{n}} \\
& =\frac{1}{\Gamma(n-\alpha)} \int_{0}^{t}(t-s)^{n-\alpha-1} u^{(n)}(s) d s,
\end{aligned}
$$

where $n$ is the smallest integer greater than or equal to $\alpha$, provided that the right-hand side integral is pointwise defined on $(0,+\infty)$. 
Lemma 2.1 (see [8]) Let $\alpha>0$. Assume that $u, D_{0^{+}}^{\alpha} u \in L([0,1], \mathbb{R})$. Then the following equality holds:

$$
I_{0^{+}}^{\alpha} D_{0^{+}}^{\alpha} u(t)=u(t)+c_{0}+c_{1} t+\cdots+c_{n-1} t^{n-1},
$$

where $c_{i} \in \mathbb{R}, i=0,1, \ldots, n-1$, and $n$ is the smallest integer greater than or equal to $\alpha$.

Lemma 2.2 For fixed $l \in C([0,1], \mathbb{R})$, let us define

$$
G_{l}(a)=\int_{0}^{1}(1-s)^{\alpha-1} \phi_{q}(a+l(s)) d s
$$

then the equation $G_{l}(a)=0$ has a unique solution $\tilde{a}(l)$, and the function $\tilde{a}: C[0,1] \rightarrow \mathbb{R}$ is continuous and sends bounded sets into bounded sets.

Proof The proof is similar to the proof of Proposition 2.2 in [35], so we omit the details.

In this paper, we take $Y=C([0,1], \mathbb{R})$ with the norm $\|y\|_{0}=\max _{t \in[0,1]}|y(t)|$, and $X=$ $\left\{x \mid x, D_{0^{+}}^{\alpha} x \in Y\right\}$ with the norm $\|x\|_{X}=\max \left\{\|x\|_{0},\left\|D_{0^{+}}^{\alpha} x\right\|_{0}\right\}$. By means of the linear functional analysis theory, we can prove that $X$ is a Banach space.

\section{Main results}

In this section, two theorems on the existence of solutions for BVP (1.2) (1.3) and BVP (1.2) (1.4) will be given under nonlinear growth restrictions of $f$.

As a consequence of Lemma 2.1, we have the following results that are useful in what follows.

Lemma 3.1 Given $h \in Y$, the unique solution of

$$
\left\{\begin{array}{l}
D_{0^{+}}^{\beta} \phi_{p}\left(D_{0^{+}}^{\alpha} x(t)\right)=h(t), \quad t \in[0,1] \\
D_{0^{+}}^{\alpha} x(0)=x(1)=0
\end{array}\right.
$$

is

$$
\begin{aligned}
x(t) & =I_{0^{+}}^{\alpha} \phi_{q}\left(I_{0^{+}}^{\beta} h\right)(t)+A h(t) \\
& =\frac{1}{\Gamma(\alpha)} \int_{0}^{t}(t-s)^{\alpha-1} \phi_{q}\left(\frac{1}{\Gamma(\beta)} \int_{0}^{s}(s-\tau)^{\beta-1} h(\tau) d \tau\right) d s+A h(t),
\end{aligned}
$$

where

$$
\begin{aligned}
A h(t) & =-I_{0^{+}}^{\alpha} \phi_{q}\left(I_{0^{+}}^{\beta} h\right)(1) \\
& =-\frac{1}{\Gamma(\alpha)} \int_{0}^{1}(1-s)^{\alpha-1} \phi_{q}\left(\frac{1}{\Gamma(\beta)} \int_{0}^{s}(s-\tau)^{\beta-1} h(\tau) d \tau\right) d s,
\end{aligned}
$$

and $\phi_{q}$ is understood as the operator $\phi_{q}: Y \rightarrow Y$ defined by $\phi_{q}(x)(t)=\phi_{q}(x(t))$. 
Proof Assume that $x(t)$ satisfies the equation of BVP (3.1), then Lemma 2.1 implies

$$
\phi_{p}\left(D_{0^{+}}^{\alpha} x(t)\right)=c_{1}+I_{0^{+}}^{\beta} h(t), \quad c_{1} \in \mathbb{R} .
$$

From the boundary value condition $D_{0^{+}}^{\alpha} x(0)=0$, one has $c_{1}=0$. Thus, we have

$$
x(t)=c_{2}+I_{0^{+}}^{\alpha} \phi_{q}\left(I_{0^{+}}^{\beta} h\right)(t), \quad c_{2} \in \mathbb{R} .
$$

By the condition $x(1)=0$, we get

$$
c_{2}=-I_{0^{+}}^{\alpha} \phi_{q}\left(I_{0^{+}}^{\beta} h\right)(1)=A h(t) .
$$

The proof is completed.

Define the operator $\mathcal{K}_{1}: X \rightarrow X$ by

$$
\begin{aligned}
\mathcal{K}_{1} x(t) & \\
= & I_{0^{+}}^{\alpha} \phi_{q}\left(I_{0^{+}}^{\beta} N_{f} x\right)(t)+A N_{f} x(t) \\
= & \frac{1}{\Gamma(\alpha)} \int_{0}^{t}(t-s)^{\alpha-1} \phi_{q}\left(\frac{1}{\Gamma(\beta)} \int_{0}^{s}(s-\tau)^{\beta-1} f\left(\tau, x(\tau), D_{0^{+}}^{\alpha} x(\tau)\right) d \tau\right) d s \\
& -\frac{1}{\Gamma(\alpha)} \int_{0}^{1}(1-s)^{\alpha-1} \phi_{q}\left(\frac{1}{\Gamma(\beta)} \int_{0}^{s}(s-\tau)^{\beta-1} f\left(\tau, x(\tau), D_{0^{+}}^{\alpha} x(\tau)\right) d \tau\right) d s
\end{aligned}
$$

where $N_{f}: X \rightarrow Y$ is the Nemytskii operator defined by

$$
N_{f} x(t)=f\left(t, x(t), D_{0^{+}}^{\alpha} x(t)\right), \quad \forall t \in[0,1]
$$

Clearly, the fixed points of $\mathcal{K}_{1}$ are the solutions of BVP (1.2) (1.3).

Lemma 3.2 Given $h \in Y$, the unique solution of

$$
\left\{\begin{array}{l}
D_{0^{+}}^{\beta} \phi_{p}\left(D_{0^{+}}^{\alpha} x(t)\right)=h(t), \quad t \in[0,1] \\
x(0)=x(1)=0
\end{array}\right.
$$

is

$$
\begin{aligned}
x(t) & =I_{0^{+}}^{\alpha} \phi_{q}\left(I_{0^{+}}^{\beta} h+B h\right)(t) \\
& =\frac{1}{\Gamma(\alpha)} \int_{0}^{t}(t-s)^{\alpha-1} \phi_{q}\left(\frac{1}{\Gamma(\beta)} \int_{0}^{s}(s-\tau)^{\beta-1} h(\tau) d \tau+B h(s)\right) d s,
\end{aligned}
$$

where

$$
\begin{aligned}
B h(t) & =-I_{0^{+}}^{\beta} h(\eta(h)) \\
& =-\frac{1}{\Gamma(\beta)} \int_{0}^{\eta(h)}(\eta(h)-s)^{\beta-1} h(s) d s,
\end{aligned}
$$

and $\eta(h) \in(0,1)$ is a unique constant dependent on $h(t)$. 
Proof Assume that $x(t)$ satisfies the equation of BVP (3.3), by Lemma 2.1, we get

$$
x(t)=c+I_{0^{+}}^{\alpha} \phi_{q}\left(a+I_{0^{+}}^{\beta} h\right)(t), \quad a, c \in \mathbb{R} .
$$

From the boundary value conditions $x(0)=x(1)=0$, one has $c=0$ and

$$
\int_{0}^{1}(1-s)^{\alpha-1} \phi_{q}\left(a+I_{0^{+}}^{\beta} h(s)\right) d s=0 .
$$

Then, basing on Lemma 2.2, we know equation (3.4) has a unique solution $\widetilde{a}\left(I_{0^{+}}^{\beta} h\right)$. Moreover, by the integral mean value theorem, there exists a unique constant $\eta(h) \in(0,1)$ dependent on $h(t)$ such that $\phi_{q}\left(\widetilde{a}\left(I_{0^{+}}^{\beta} h\right)+I_{0^{+}}^{\beta} h\right)(\eta(h))=0$, which implies $\left(\widetilde{a}\left(I_{0^{+}}^{\beta} h\right)+I_{0^{+}}^{\beta} h\right)(\eta(h))=$ 0 . Thus, we have

$$
\widetilde{a}\left(I_{0^{+}}^{\beta} h\right)=-I_{0^{+}}^{\beta} h(\eta(h))=B h(t) .
$$

The proof is completed.

Define the operator $\mathcal{K}_{2}: X \rightarrow X$ by

$$
\begin{aligned}
\mathcal{K}_{2} x(t) & \\
= & I_{0^{+}}^{\alpha} \phi_{q}\left(I_{0^{+}}^{\beta} N_{f} x+B N_{f} x\right)(t) \\
= & \frac{1}{\Gamma(\alpha)} \int_{0}^{t}(t-s)^{\alpha-1} \phi_{q}\left(\frac{1}{\Gamma(\beta)} \int_{0}^{s}(s-\tau)^{\beta-1} f\left(\tau, x(\tau), D_{0^{+}}^{\alpha} x(\tau)\right) d \tau\right. \\
& \left.-\frac{1}{\Gamma(\beta)} \int_{0}^{\eta(x)}(\eta(x)-\tau)^{\beta-1} f\left(\tau, x(\tau), D_{0^{+}}^{\alpha} x(\tau)\right) d \tau\right) d s,
\end{aligned}
$$

where $\eta(x) \in(0,1)$ is a unique constant dependent on $x(t)$ and $N_{f}$ is the Nemytskii operator defined by (3.2). Clearly, the fixed points of $\mathcal{K}_{2}$ are the solutions of BVP (1.2) (1.4).

Our first result, based on Schaefer's fixed point theorem and Lemma 3.1, is stated as follows.

Theorem 3.1 Let $f:[0,1] \times \mathbb{R}^{2} \rightarrow \mathbb{R}$ be continuous. Assume that

$(\mathrm{H})$ there exist nonnegative functions $a, b, c \in Y$ such that

$$
|f(t, u, v)| \leq a(t)+b(t)|u|^{p-1}+c(t)|v|^{p-1}, \quad \forall t \in[0,1],(u, v) \in \mathbb{R}^{2}
$$

Then BVP (1.2) (1.3) has at least one solution provided that

$$
\gamma_{1}:=\frac{1}{\Gamma(\beta+1)}\left[\frac{2^{p-1}\|b\|_{0}}{(\Gamma(\alpha+1))^{p-1}}+\|c\|_{0}\right]<1 .
$$

Proof The proof will be given in the following two steps.

Step 1: $\mathcal{K}_{1}: X \rightarrow X$ is completely continuous.

By the definition of $\mathcal{K}_{1}$, we obtain

$$
D_{0^{+}}^{\alpha} \mathcal{K}_{1} x(t)=\phi_{q}\left(I_{0^{+}}^{\beta} N_{f} x\right)(t)
$$


Obviously, the operators $\mathcal{K}_{1}$ and $D_{0^{+}}^{\alpha} \mathcal{K}_{1}$ are compositions of the continuous operators. So $\mathcal{K}_{1}$ and $D_{0^{+}}^{\alpha} \mathcal{K}_{1}$ are continuous in $Y$. Hence, $\mathcal{K}_{1}$ is a continuous operator.

Let $\Omega \subset X$ be an open bounded set, then $\mathcal{K}_{1}(\bar{\Omega})$ and $D_{0^{+}}^{\alpha} \mathcal{K}_{1}(\bar{\Omega})$ are bounded. Moreover, for $\forall x \in \bar{\Omega}, t \in[0,1]$, there exists a constant $T>0$ such that $\left|I_{0^{+}}^{\beta} N_{f} x(t)\right| \leq T$. Thus, in view of the Arzelà-Ascoli theorem, we need only to prove that $\mathcal{K}_{1}(\bar{\Omega}) \subset X$ is equicontinuous.

For $0 \leq t_{1}<t_{2} \leq 1, x \in \bar{\Omega}$, we have

$$
\begin{aligned}
& \left|\mathcal{K}_{1} x\left(t_{2}\right)-\mathcal{K}_{1} x\left(t_{1}\right)\right| \\
& \quad=\frac{1}{\Gamma(\alpha)}\left|\int_{0}^{t_{2}}\left(t_{2}-s\right)^{\alpha-1} \phi_{q}\left(I_{0^{+}}^{\beta} N_{f} x(s)\right) d s-\int_{0}^{t_{1}}\left(t_{1}-s\right)^{\alpha-1} \phi_{q}\left(I_{0^{+}}^{\beta} N_{f} x(s)\right) d s\right| \\
& \quad \leq \frac{T^{q-1}}{\Gamma(\alpha)}\left\{\int_{0}^{t_{1}}\left[\left(t_{1}-s\right)^{\alpha-1}-\left(t_{2}-s\right)^{\alpha-1}\right] d s+\int_{t_{1}}^{t_{2}}\left(t_{2}-s\right)^{\alpha-1} d s\right\} \\
& =\frac{T^{q-1}}{\Gamma(\alpha+1)}\left[t_{1}^{\alpha}-t_{2}^{\alpha}+2\left(t_{2}-t_{1}\right)^{\alpha}\right] .
\end{aligned}
$$

Since $t^{\alpha}$ is uniformly continuous in $[0,1]$, we can obtain that $\mathcal{K}_{1}(\bar{\Omega}) \subset Y$ is equicontinuous. A similar proof can show that $I_{0^{+}}^{\beta} N_{f}(\bar{\Omega}) \subset Y$ is equicontinuous. This, together with the uniform continuity of $\phi_{q}(s)$ on $[-T, T]$, yields that $D_{0^{+}}^{\alpha} \mathcal{K}_{1}(\bar{\Omega}) \subset Y$ is also equicontinuous. Thus, we find that $\mathcal{K}_{1}: X \rightarrow X$ is compact.

Step 2: A priori bounds.

Set

$$
\Omega_{1}=\left\{x \in X \mid x=\lambda^{q-1} \mathcal{K}_{1} x, \lambda \in(0,1)\right\} .
$$

Now it remains to show that the set $\Omega_{1}$ is bounded.

By Lemma 2.1 and the boundary value condition $x(1)=0$, one has

$$
x(t)=I_{0_{+}}^{\alpha} D_{0^{+}}^{\alpha} x(t)-I_{0_{+}}^{\alpha} D_{0^{+}}^{\alpha} x(1),
$$

which together with

$$
\begin{aligned}
\left|I_{0^{+}}^{\alpha} D_{0^{+}}^{\alpha} x(t)\right| & =\frac{1}{\Gamma(\alpha)}\left|\int_{0}^{t}(t-s)^{\alpha-1} D_{0^{+}}^{\alpha} x(s) d s\right| \\
& \leq \frac{1}{\Gamma(\alpha)}\left\|D_{0^{+}}^{\alpha} x\right\|_{0} \cdot \frac{1}{\alpha} t^{\alpha} \\
& \leq \frac{1}{\Gamma(\alpha+1)}\left\|D_{0^{+}}^{\alpha} x\right\|_{0^{\prime}}, \quad \forall t \in[0,1]
\end{aligned}
$$

yields

$$
\|x\|_{0} \leq \frac{2}{\Gamma(\alpha+1)}\left\|D_{0^{+}}^{\alpha} x\right\|_{0} .
$$

For $x \in \Omega_{1}$, we have

$$
\begin{aligned}
\phi_{p}\left(D_{0^{+}}^{\alpha} x(t)\right) & =\lambda I_{0^{+}}^{\beta} N_{f} x(t) \\
& =\frac{\lambda}{\Gamma(\beta)} \int_{0}^{t}(t-s)^{\beta-1} f\left(s, x(s), D_{0^{+}}^{\alpha} x(s)\right) d s .
\end{aligned}
$$


So, from $(\mathrm{H})$, we obtain

$$
\begin{aligned}
& \left|\phi_{p}\left(D_{0^{+}}^{\alpha} x(t)\right)\right| \\
& \quad \leq \frac{1}{\Gamma(\beta)} \int_{0}^{t}(t-s)^{\beta-1}\left(a(s)+b(s)|x(s)|^{p-1}+c(s)\left|D_{0^{+}}^{\alpha} x(s)\right|^{p-1}\right) d s \\
& \quad \leq \frac{1}{\Gamma(\beta)}\left(\|a\|_{0}+\|b\|_{0}\|x\|_{0}^{p-1}+\|c\|_{0}\left\|D_{0^{+}}^{\alpha} x\right\|_{0}^{p-1}\right) \cdot \frac{1}{\beta} t^{\beta} \\
& \quad \leq \frac{1}{\Gamma(\beta+1)}\left(\|a\|_{0}+\|b\|_{0}\|x\|_{0}^{p-1}+\|c\|_{0}\left\|D_{0^{+}}^{\alpha} x\right\|_{0}^{p-1}\right), \quad \forall t \in[0,1],
\end{aligned}
$$

which, together with $\left|\phi_{p}\left(D_{0^{+}}^{\alpha} x(t)\right)\right|=\left|D_{0^{+}}^{\alpha} x(t)\right|^{p-1}$ and (3.7), yields

$$
\left\|D_{0^{+}}^{\alpha} x\right\|_{0}^{p-1} \leq \frac{1}{\Gamma(\beta+1)}\left[\|a\|_{0}+\left(\frac{2^{p-1}\|b\|_{0}}{(\Gamma(\alpha+1))^{p-1}}+\|c\|_{0}\right)\left\|D_{0^{+}}^{\alpha} x\right\|_{0}^{p-1}\right] .
$$

Hence, in view of (3.5), we can get

$$
\left\|D_{0^{+}}^{\alpha} x\right\|_{0} \leq\left[\frac{\|a\|_{0}}{\left(1-\gamma_{1}\right) \Gamma(\beta+1)}\right]^{q-1}:=M_{11}
$$

Thus, from (3.7), one has

$$
\|x\|_{0} \leq \frac{2 M_{11}}{\Gamma(\alpha+1)}:=M_{12}
$$

Therefore, combining (3.8) with (3.9), we have

$$
\begin{aligned}
\|x\|_{X} & =\max \left\{\|x\|_{0},\left\|D_{0^{+}}^{\alpha} x\right\|_{0}\right\} \\
& \leq \max \left\{M_{11}, M_{12}\right\} .
\end{aligned}
$$

As a consequence of Schaefer's fixed point theorem, we deduce that $\mathcal{K}_{1}$ has a fixed point which is the solution of BVP (1.2) (1.3). The proof is completed.

Our second result, based on Schaefer's fixed point theorem and Lemma 3.2, is stated as follows.

Theorem 3.2 Let $f:[0,1] \times \mathbb{R}^{2} \rightarrow \mathbb{R}$ be continuous. Suppose $(\mathrm{H})$ holds, then BVP (1.2) (1.4) has at least one solution provided that

$$
\gamma_{2}:=\frac{2}{\Gamma(\beta+1)}\left[\frac{\|b\|_{0}}{(\Gamma(\alpha+1))^{p-1}}+\|c\|_{0}\right]<1
$$

Proof The proof of complete continuity of $\mathcal{K}_{2}$ is similar to the proof of complete continuity of $\mathcal{K}_{1}$, so we omit the details.

Set

$$
\Omega_{2}=\left\{x \in X \mid x=\lambda^{q-1} \mathcal{K}_{2} x, \lambda \in(0,1)\right\} .
$$


From Lemma 2.1 and $x(0)=0$, one has

$$
x(t)=I_{0_{+}}^{\alpha} D_{0^{+}}^{\alpha} x(t),
$$

which together with (3.6) yields

$$
\|x\|_{0} \leq \frac{1}{\Gamma(\alpha+1)}\left\|D_{0^{+}}^{\alpha} x\right\|_{0}
$$

Then, by the hypothesis $(\mathrm{H})$ and (3.11), we obtain

$$
\begin{aligned}
& \left|I_{0^{+}}^{\beta} N_{f} x(t)\right| \leq \frac{1}{\Gamma(\beta+1)}\left[\left(\frac{\|b\|_{0}}{(\Gamma(\alpha+1))^{p-1}}+\|c\|_{0}\right)\left\|D_{0^{+}}^{\alpha} x\right\|_{0}^{p-1}+\|a\|_{0}\right] \\
& \quad \forall t \in[0,1] .
\end{aligned}
$$

For $x \in \Omega_{2}$, we have

$$
\phi_{p}\left(D_{0^{+}}^{\alpha} x(t)\right)=\lambda I_{0^{+}}^{\beta} N_{f} x(t)-\lambda I_{0^{+}}^{\beta} N_{f} x(\eta(x)), \quad \eta(x) \in(0,1),
$$

which, together with $\left|\phi_{p}\left(D_{0^{+}}^{\alpha} x(t)\right)\right|=\left|D_{0^{+}}^{\alpha} x(t)\right|^{p-1}$ and (3.12), yields

$$
\left\|D_{0^{+}}^{\alpha} x\right\|_{0}^{p-1} \leq \frac{2}{\Gamma(\beta+1)}\left[\|a\|_{0}+\left(\frac{\|b\|_{0}}{(\Gamma(\alpha+1))^{p-1}}+\|c\|_{0}\right)\left\|D_{0^{+}}^{\alpha} x\right\|_{0}^{p-1}\right]
$$

Thus, in view of (3.10), we can get

$$
\left\|D_{0^{+}}^{\alpha} x\right\|_{0} \leq\left[\frac{2\|a\|_{0}}{\left(1-\gamma_{2}\right) \Gamma(\beta+1)}\right]^{q-1}:=M_{21} .
$$

So, from (3.11), one has

$$
\|x\|_{0} \leq \frac{M_{21}}{\Gamma(\alpha+1)}:=M_{22}
$$

Hence, combining (3.13) with (3.14), we obtain

$$
\|x\|_{X} \leq \max \left\{M_{21}, M_{22}\right\} .
$$

Therefore, $\Omega_{2}$ is bounded.

As a consequence of Schaefer's fixed point theorem, we deduce that $\mathcal{K}_{2}$ has a fixed point which is the solution of BVP (1.2) (1.4). The proof is completed.

\section{An example}

In this section, we give an example to illustrate our main results.

Example 4.1 Consider the following fractional $p$-Laplacian equation:

$$
D_{0^{+}}^{\frac{3}{4}} \phi_{3}\left(D_{0^{+}}^{\frac{1}{2}} x(t)\right)=\sin t+\frac{1}{6} x^{2}(t)+t \cos \left(D_{0^{+}}^{\frac{1}{2}} x(t)\right), \quad t \in[0,1]
$$


Corresponding to equation (1.2), we get $p=3, \alpha=1 / 2, \beta=3 / 4$ and

$$
f(t, u, v)=\sin t+\frac{1}{6} u^{2}+t \cos v
$$

Choose $a(t)=2, b(t)=1 / 6, c(t)=0$. By a simple calculation, we obtain $\|b\|_{0}=1 / 6,\|c\|_{0}=0$ and

$$
\begin{aligned}
& \gamma_{1}=\frac{1}{\Gamma\left(\frac{3}{4}+1\right)}\left[\frac{2^{2}}{\left(\Gamma\left(\frac{1}{2}+1\right)\right)^{2}} \times \frac{1}{6}+0\right]<1, \\
& \gamma_{2}=\frac{2}{\Gamma\left(\frac{3}{4}+1\right)}\left[\frac{1}{\left(\Gamma\left(\frac{1}{2}+1\right)\right)^{2}} \times \frac{1}{6}+0\right]<1 .
\end{aligned}
$$

Obviously, equation (4.1) subject to the boundary value conditions (1.3) (or (1.4)) satisfies all assumptions of Theorem 3.1 (or Theorem 3.2). Hence, BVP (4.1) (1.3) (or BVP (4.1) (1.4)) has at least one solution.

\section{Competing interests}

The author declares that he has no competing interests.

\section{Author's contributions}

The author contributed to the manuscript. The author read and approved the final manuscript.

\section{Acknowledgements}

The author is grateful for the valuable comments and suggestions of the referees.

Received: 20 July 2015 Accepted: 27 September 2015 Published online: 17 November 2015

\section{References}

1. Agarwal, RP, Belmekki, M, Benchohra, M: A survey on semilinear differential equations and inclusions involving Riemann-Liouville fractional derivative. Adv. Differ. Equ. 2009, Article ID 981728 (2009)

2. Agarwal, RP, Benchohra, M, Hamani, S: Boundary value problems for fractional differential equations. Georgian Math. J. 16, 401-411 (2009)

3. Agarwal, RP, Benchohra, M, Hamani, S: A survey on existence results for boundary value problems of nonlinear fractional differential equations and inclusions. Acta Appl. Math. 109, 973-1033 (2010)

4. Delbosco, D, Rodino, L: Existence and uniqueness for a nonlinear fractional differential equation. J. Math. Anal. Appl. 204, 609-625 (1996)

5. He, JH: Approximate analytical solution for seepage flow with fractional derivatives in porous media. Comput. Methods Appl. Mech. Eng. 167, 57-58 (1998)

6. He, JH: Some applications of nonlinear fractional differential equations and their approximations. Bull. Sci. Technol. $15,86-90(1999)$

7. Jaradat, OK, Al-Omari, A, Momani, S: Existence of mild solutions for fractional semilinear initial value problems. Nonlinear Anal. 69, 3153-3159 (2008)

8. Kilbas, AA, Srivastava, HM, Trujillo, Jj: Theory and Applications of Fractional Differential Equations. Elsevier, Amsterdam (2006)

9. Babakhani, A, Gejji, VD: Existence of positive solutions of nonlinear fractional differential equations. J. Math. Anal. Appl. 278, 434-442 (2003)

10. Kilbas, AA, Trujillo, JJ: Differential equations of fractional order: methods, results and problems-I. Appl. Anal. 78, 153-192 (2001)

11. Kilbas, AA, Trujillo, JJ: Differential equations of fractional order: methods, results and problems-ll. Appl. Anal. 81, 435-493 (2002)

12. Agarwal, RP, O'Regan, D, Stanek, S: Positive solutions for Dirichlet problems of singular nonlinear fractional differential equations. J. Math. Anal. Appl. 371, 57-68 (2010)

13. Bai, Z: On solutions of some fractional $m$-point boundary value problems at resonance. Electron. J. Qual. Theory Differ. Equ. 2010, 37 (2010)

14. Chen, T, Liu, W, Hu, Z: A boundary value problem for fractional differential equation with $p$-Laplacian operator at resonance. Nonlinear Anal. 75, 3210-3217 (2012)

15. Chen, T, Liu, W: An anti-periodic boundary value problem for the fractional differential equation with a $p$-Laplacian operator. Appl. Math. Lett. 25, 1671-1675 (2012)

16. Benchohra, M, Hamani, S, Ntouyas, SK: Boundary value problems for differential equations with fractional order and nonlocal conditions. Nonlinear Anal. 71, 2391-2396 (2009)

17. Bai, Z, Lü, H: Positive solutions for boundary value problem of nonlinear fractional differential equation. J. Math. Anal. Appl. 311, 495-505 (2005) 
18. Darwish, MA, Ntouyas, SK: On initial and boundary value problems for fractional order mixed type functional differential inclusions. Comput. Math. Appl. 59, 1253-1265 (2010)

19. El-Shahed, M, Nieto, JJ: Nontrivial solutions for a nonlinear multi-point boundary value problem of fractional order. Comput. Math. Appl. 59, 3438-3443 (2010)

20. Jiang, W: The existence of solutions to boundary value problems of fractional differential equations at resonance. Nonlinear Anal. 74, 1987-1994 (2011)

21. Kosmatov, N: A boundary value problem of fractional order at resonance. Electron. J. Differ. Equ. 2010, 135 (2010)

22. Su, X: Boundary value problem for a coupled system of nonlinear fractional differential equations. Appl. Math. Lett. $22,64-69(2009)$

23. Leibenson, LS: General problem of the movement of a compressible fluid in a porous medium. Izv. Akad. Nauk Kirg. SSSR 9, 7-10 (1983) (in Russian)

24. Chen, T, Liu, W, Yang, C: Antiperiodic solutions for Liénard-type differential equation with $p$-Laplacian operator. Bound. Value Probl. 2010, Article ID 194824 (2010)

25. Chen, T, Liu, W, Hu, Z: New results on the existence of periodic solutions for a higher-order Liénard type $p$-Laplacian differential equation. Math. Methods Appl. Sci. 34, 2189-2196 (2011)

26. Jiang, D, Gao, W: Upper and lower solution method and a singular boundary value problem for the one-dimensional p-Laplacian. J. Math. Anal. Appl. 252, 631-648 (2000)

27. Lian, $L, G e, W$ : The existence of solutions of $m$-point $p$-Laplacian boundary value problems at resonance. Acta Math. Appl. Sin. 28, 288-295 (2005)

28. Liu, B, Yu, J: On the existence of solutions for the periodic boundary value problems with $p$-Laplacian operator. J. Syst. Sci. Math. Sci. 23, 76-85 (2003)

29. Pang, $\mathrm{H}, \mathrm{Ge}, \mathrm{W}$, Tian, M: Solvability of nonlocal boundary value problems for ordinary differential equation of higher order with a $p$-Laplacian. Comput. Math. Appl. 56, 127-142 (2008)

30. Su, H, Wang, B, Wei, Z, Zhang, X: Positive solutions of four-point boundary value problems for higher-order p-Laplacian operator. J. Math. Anal. Appl. 330, 836-851 (2007)

31. Zhang, J, Liu, W, Ni, J, Chen, T: Multiple periodic solutions of $p$-Laplacian equation with one-side Nagumo condition. J. Korean Math. Soc. 45, 1549-1559 (2008)

32. Miller, KS, Ross, B: An Introduction to the Fractional Calculus and Fractional Differential Equations. Wiley, New York (1993)

33. Podlubny, I: Fractional Differential Equation. Academic Press, San Diego (1999)

34. Samko, SG, Kilbas, AA, Marichev, OI: Fractional Integrals and Derivatives: Theory and Applications. Gordon \& Breach, Yverdon (1993)

35. Manásevich, R, Mawhin, J: Periodic solutions for nonlinear systems with p-Laplacian-like operators. J. Differ. Equ. 145, 367-393 (1998)

\section{Submit your manuscript to a SpringerOpen ${ }^{\odot}$ journal and benefit from:}

$\checkmark$ Convenient online submission

- Rigorous peer review

Immediate publication on acceptance

Open access: articles freely available online

- High visibility within the field

- Retaining the copyright to your article 